Radio Interferometry: Theory, Techniques and Applications,

IAU Coll. 131, ASP Conference Series, Vol. 19.1991,

T.J. Comwell and R.A. Perley (eds.)

USING SUB-APERTURE ILLUMINATION TO SOLVE THE SHORTSPACING PROBLEM

\author{
DAVID P. WOODY and NICK Z. SCOVILLE \\ Owens Valley Radio Observatory, California Institute of Technology, \\ PO Box 968, Big Pine, Ca 93513
}

LEE G. MUNDY

University of Maryland, Astronomy Program, College Park, MD 20742

\begin{abstract}
A common problem in producing interferometer maps of objects with structure on the scale-size of the primary beam is the acquisition of UV data corresponding to telescope spacings of less than one telescope diameter. One technique for solving this "short-spacing" problem is to divide the aperture of one of the telescopes into smaller sub-apertures and measuring the visibilities between these sub-apertures. This technique is being tested using the Owens Valley Millimeter Interferometer.
\end{abstract}

\title{
INTRODUCTION
}

Many of the objects of interest at millimeter-wave wavelengths are many arcminutes across. This presents a problem for synthesis mapping using an interferometer array of uniformly sized antennas, since the objects are often larger than the primary beam of the single elements in the interferometer. The problem arises because the shortest nominal spacing in the UV plane sampled by an interferometer is $\mathrm{D}$, where $\mathrm{D}$ is the antenna diameter. A single measurement samples the UV plane corresponding to zero spacing. Thus the region at a distance $\mathrm{D} / 2$ from the center of the UV plane is poorly sampled. The observed visibilities are actually the true visibilities convolved with the UV spectral response of the system (Thompson, Moran and Swenson 1986). The UV spectral response is the voltage cross correlation for the aperture fields of the two antennas and has a maximum width of $2 \mathrm{D}$ centered at the UV position corresponding to the antenna separation. Similarly, the UV spectral response of a single antenna is the autocorrelation of its aperture field pattern and also has a maximum width of $2 \mathrm{D}$. The crossing point for the spectral responses for a pair of interferometer antennas touching at their edge and for a single dish is D/2 from the center of the UV-plane. For circular antennas with Gaussian illumination tapered to $13 \mathrm{~dB}$ at the edge with $2 \%$ aperture blockage, the spectral responses overlap at the $35 \%$ level. This increases to $45 \%$ for uniform illumination with $2 \%$ blockage. 


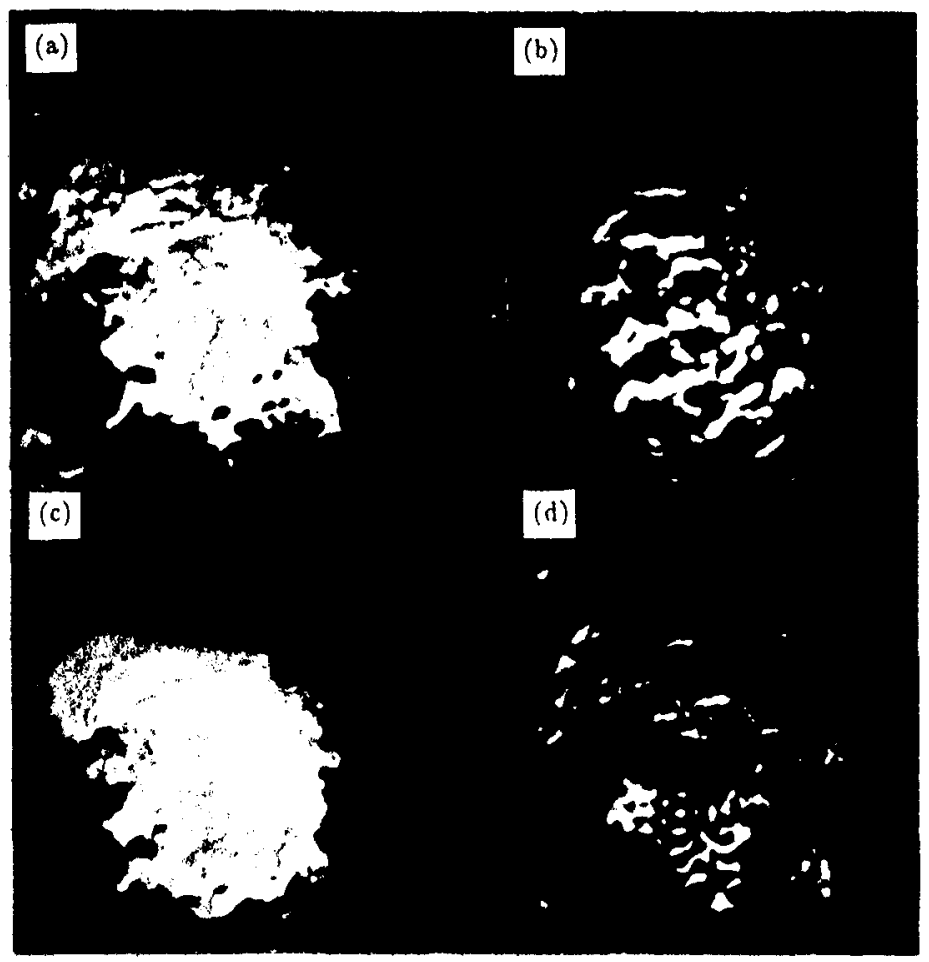

Fig. 1. Simulation of image reconstruction using sub-apertures. Panel (a) is the model source. (b) shows the reconstructed image made using only the full aperture interferometer mosaic data. (c) is the reconstructed image made using the whole data set including the sub-aperture and single dish observations. (d) shows the reconstruction errors, i.e. (a) minus (c).

The usual method for obtaining the D/2 UV data is to combine the interferometer data with maps made using a single dish of diameter $>2 \mathrm{D}$. It has also been proposed that the visibilities around the $\mathrm{D} / 2 \mathrm{UV}$ spacing can be recovered using deconvolution techniques on mosaic maps (Ekers and Rots, 1979). This involves mapping the source at the Nyquist sampling of $\lambda / 2 \mathrm{D}$ on the sky using both the interferometer and the single dish systems. Simulations of this technique using the proposed 40 element NRAO millimeter array, MMA, are presented by Holdaway (these proceedings). This paper presents a new method for obtaining the visibilities on a circle of radius $\mathrm{D} / 2$ in the $U V$-plane.

\section{SUB-APERTURE SIMULATION}

Another approach to obtaining the short-spacing visibilities is to divide the aperture of an antenna into two smaller sub-apertures whose diameter is half that of the full aperture. This yields the D/2 UV data directly when the 
visibility between the sub-apertures is observed. A full circle at radius $D / 2$ in the UV plane can be obtained by observing with an alt-az mount for six hours or by using a rotator to sweep the sub-apertures around the optical axis of the antenna. Additionally the visibilities measured between the sub-apertures on one antenna and the other antennas in the array gives higher resolution UV data from $D / 2$ outward.

The combination of the array interferometer data, sub-aperture data and single dish data from the array dishes can be used to produce maps covering scale sizes from one arcsec to more than one degree. We have made simulated maps from this combined data set using an MEM package written by Tim Cornwell of NRAO. A $2 \mathrm{~cm}$ VLA map of M42 was used as a test model. Panel (a) in fig. 1 shows the model source and (b) shows the reconstructed image made using only the full aperture interferometer mosaic map. As expected, the extended flux is missing. Panel (c) is the reconstructed image made using the whole data set including the sub-aperture and single dish observations. This image recovers most of the information. The difference between the model map and the reconstructed image is presented in panel (d).

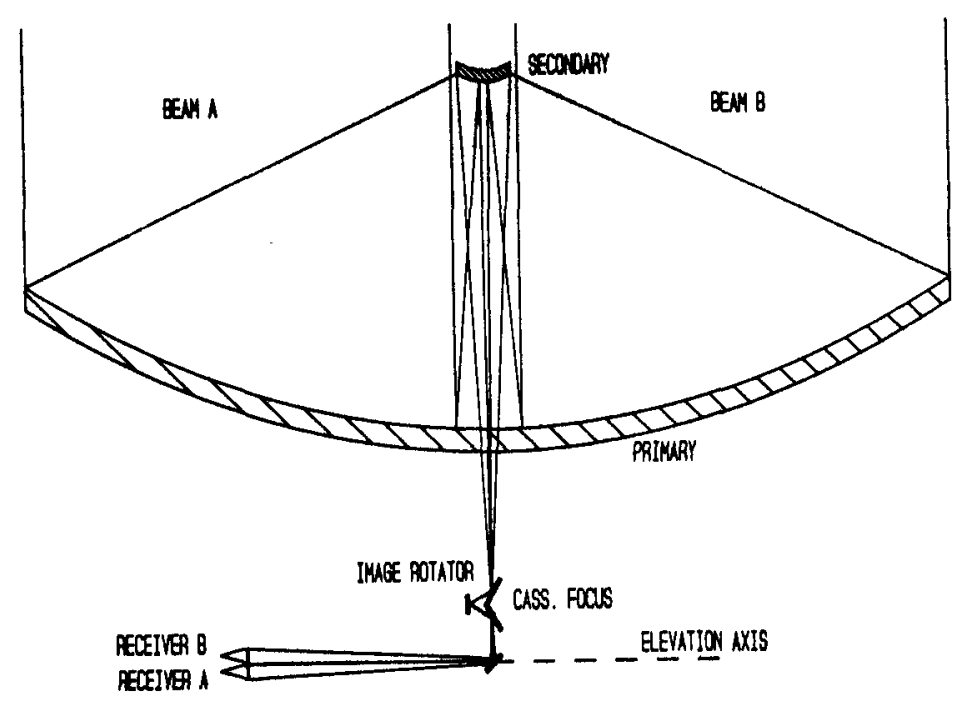

Fig. 2. Schematic drawing of the sub-aperture optics.

\section{OPTICS}

The concept of using sub-apertures to obtain the D/2 UV spacing visibility data is being tested on the OVRO Millimeter Array. The Nasmyth optics makes it convenient to use two receivers to illuminate the 10.4 meter primary with independent 5 meter diameter patches. A schematic diagram of the optics is shown in figure 2. The receiver cryostat is mounted on a plate which is bolted to an extension of the telescopes elevation axis in the receiver room. The cryostat has two windows separated by five inches for the two receivers. 
An off-axis ellipsoid is mounted above each receiver to refocus and direct their beams to the Cassegrain focus position and then onto separate halves of the primary. A three mirror image rotator at the Cassegrain focus will be used to rotate the position of the sub-apertures about the telescope's optical axis. This will allow us to provide more UV coverage in combination with the neighboring telescopes and to cover the full $\mathrm{D} / 2 \mathrm{UV}$ circle in less than six hours. The combination of normal interferometer data, sub-aperture visibilities and singledish maps can be used to provide full imaging on all scale sizes larger than 1 arcsec.

\section{$\underline{\text { RESULTS }}$}

A prototype sub-aperture system was installed on one of the array telescopes during the summer of 1990 to evaluate its performance. The system produced the expected fringe magnitudes on the planets Venus, Mars and Jupiter but suffered from a large cross-correlation signal which was not of astronomical origin.

One of the primary difficulties encountered with using sub-apertures is the false fringes caused by telescope emission from the areas where the two beams overlap. The astronomical sources of interest are usually quite weak and our design goal is keep the false fringes below $1 \mathrm{Jy}$. This corresponds to less than $10 \mathrm{mK}$ of correlated radiation received by the two receivers for the $5 \mathrm{~m}$ diameter sub-apertures. The design to achieve this level of performance required that the two illumination functions overlap at the $-20 \mathrm{~dB}$ level. Unfortunately the feed pattern for our $2.6 \mathrm{~mm}$ wavelength receivers were measured to have sidelobes significantly larger than the desired gaussian pattern. This resulted in larger than expected overlap and produced false fringes many times larger than expected.

The interferometer cannot distinguish this false fringe from the astronomical visibilities. The false fringe is stable and thus can be measured off-source and subtracted from the data. Another method is to use the telescope to sweep the sub-aperture interferometer beam across the source and use only the modulated part of the observed correlator output.

We plan on improving the receiver feed pattern and implementing a procedure for subtracting the false fringes from the correlator output. This should give the array the capability of producing useable images covering scale sizes from one arcsec to larger than one degree.

\section{REFERENCES}

Ekers, R. D. and Rots R. A. 1979, Proceedings of IAU Colloquium No. 49, Image Formation from Coherence Functions in Astronomy, ed. C. van Schooneveld; publ. Reidel, Boston pp. 61-66.

Holdaway, M., in this proceedings.

Thompson, A. R., Moran, J. M., and Swenson, G. W. 1986, Interferometry and Synthesis in Radio Astronomy, John Wiley and Sons, New York. 
R. Ekers: The Fourier components you extract using the subaperture illumination are the same Fourier components you could extract from an image made by scanning the single dish with full aperture illumination. The question then is what is the advantage of this method?

Dave Woody: This method yields the $\mathrm{D} / 2$ uv spacing data more directly without some of the errors associated with single dish flux measurements in the presence of variable sky emission or deconvolution errors resulting from beam shape uncertainties or noise in the visibility data.

Colin Masson: Can you say something about the effect of pointing errors on this technique?

David Woody: To first order pointing errors give phase errors in the measured visibilities and hence will limit your dynamic range. It turns out that the pointing requirements are very similar to the requirements for recovering images from mosaic maps, i.e. $\leq 1 / 20$ of a beam. 